\title{
Factibilidad de uso del PET reciclado en elementos de cubiertas y envolventes
}

\section{Feasibility of use of recycled PET on ceilings and wall coverings}

\author{
Lizeth Rodríguez Rodríguez \\ lrrodriguez@uca.edu.sv \\ Docente Investigadora, Departamento de Organización del Espacio DOE-UCA \\ Mario Wilfredo Chávez Molina \\ mchavez@uca.edu.sv \\ Docente Investigador, Departamento de Ciencias Energéticas y Fluídicas DCEF-UCA \\ Florencia Lartategui de Roshardt \\ flartategui@uca.edu.sv \\ Docente Investigadora, Departamento de Mecánica Estructural DME-UCA. \\ Ana del Pilar Letona Álvarez. \\ aletona@uca.edu.sv \\ Docente Investigadora, Departamento de Operaciones y Sistemas DOS-UCA.
}

Recibido: 21 de noviembre 2016

Aceptado: 8 de febrero 2017

\section{Resumen}

En términos cognitivos, este proyecto de investigación busca responder a esta pregunta central: “¿Cuáles son los procesos de transformación no industrializado y qué características debe poseer el plástico PET reciclado para que sea reutilizado cómo materia prima para la producción de nuevos materiales de construcción?".

Para intentar responder la pregunta de investigación, se parte de la hipótesis central: que sí es posible controlar los parámetros que inciden en el proceso de transformación no industrializado del plástico PET reciclado para garantizar que las propiedades físicas cumplan con los parámetros que son requeridos a los materiales de construcción para cubierta de techo y recubrimiento en paredes. La comprobación de esta hipótesis requiere de nuevos enfoques de investigación interdisciplinar en pro del estudio de características físicas y operativas propias del material para verificar, así, la factibilidad de convertirse en material de construcción en el contexto salvadoreño.

Palabras clave

Reciclaje, materiales, desechos, plásticos, cubiertas. 


\begin{abstract}
This research project aims to answer the following question: Which is the non-industrialized transformation process and what characteristics must the PET plastic have in order to be reused as a raw material in the production of new building materials?

To answer this, the following hypothesis was taken into account: that it is possible to control the parameters that influence the non-industrialized transformation process of the PET recycled plastic in order to make the physical properties meet the required parameters of building materials for ceiling and wall coverings. The verification of this hypothesis requires new interdisciplinary approaches that favor the study of physical and operative characteristics of the material in order to verify the feasibility of becoming a building material in the Salvadorian context.
\end{abstract}

Keywords

Recycling, materials, waste, plastics, ceilings. 


\section{INTRODUCCIÓN}

La Universidad Centroamericana José Simeón Cañas UCA de El Salvador lanza anualmente una convocatoria para concursar por fondos de investigación, que busca estimular y fortalecer las capacidades investigativas de sus académicos y, a partir de ahí, incidir con mayor fuerza en los temas clave de la realidad salvadoreña y centroamericana. En este sentido, se apoyaron proyectos que estaban en sintonía con las líneas de investigación de la UCA, cuya duración no fuese mayor a un año y que, prioritariamente, suponían el trabajo articulado de varias unidades académicas. En este contexto se formula el proyecto de investigación: "Sistema constructivo para cubiertas y envolventes livianos de baja conductividad térmica derivados del reciclaje de plásticos", que consiste en el estudio de factibilidad para la utilización del plástico PET en el diseño y desarrollo de materiales alternativos para cubiertas de techo y revestimiento en paredes, a partir del estudio experimental en laboratorio sobre las características mecánicas y térmicas del polímero reciclado.
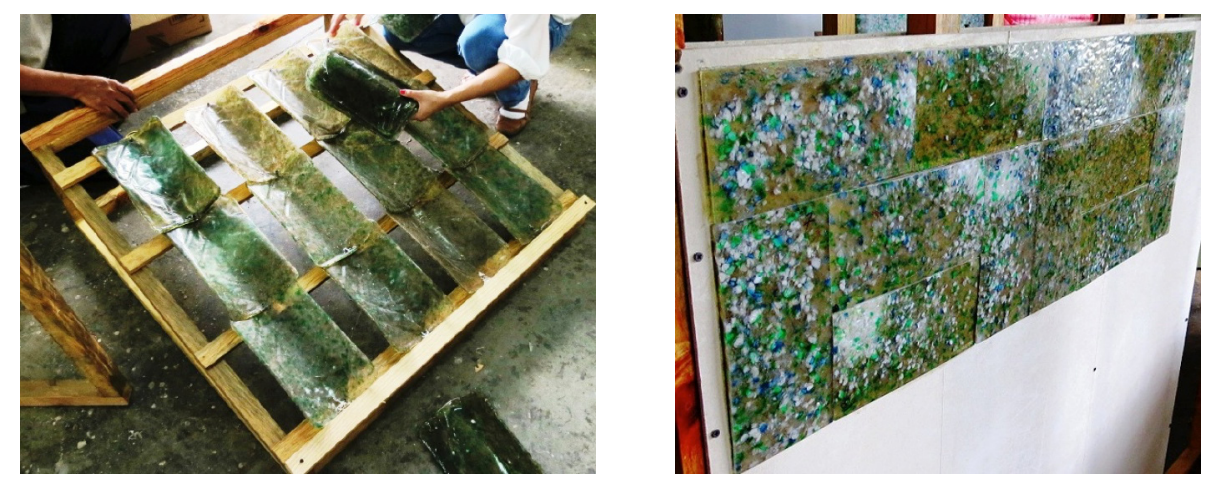

Figura 1 Modelos a escala de un metro cuadrado de techo cubierto con teja de PET reciclado y un metro cuadrado de superficie de fibrocemento revestida con baldosas de PET reciclado.

El proyecto de investigación busca responder a la pregunta de investigación central: “Cuáles son los procesos de transformación no industrializado y qué características debe poseer el plástico PET reciclado para que sea reutilizado cómo materia prima para la producción de nuevos materiales de construcción?".

Para intentar responder la pregunta de investigación, se parte de la hipótesis central: que sí es posible controlar los parámetros que inciden en el proceso de transformación no industrializado del plástico PET reciclado para garantizar que las propiedades físicas cumplan con los parámetros que son requeridos a los materiales de construcción para cubierta de techo y recubrimiento en paredes (1).

La comprobación de esta hipótesis requiere de nuevos enfoques de investigación interdisciplinar en pro del estudio de características físicas y operativas propias del material y verificar, así, la factibilidad de convertirse en material de construcción en el contexto salvadoreño.

Tomando en cuenta este contexto salvadoreño, se estima que la generación de 
desechos sólidos urbanos en El Salvador de acuerdo al dato promedio de generación per cápita para 1977 era de $0.55 \mathrm{~kg} / \mathrm{hab} /$ día; para 1997 era de $0.75 \mathrm{~kg} / \mathrm{hab} /$ día, sufriendo un incremento del 36\%; y para el año 2010 era de $0.89 \mathrm{~kg}$ /habitante/día, contemplando un incremento del $18 \%$ (2). El crecimiento en la generación de desechos por habitante es relativamente constante; sin embargo, la tipología de desecho y su composición no ha sido la misma desde hace más de treinta y cinco años. Una causa es que el estilo de vida actual, sobre todo en la ciudad, ha sido influenciado por fenómenos sociales y mercadológicos que modifican los hábitos de consumo de los ciudadanos; otra causa se debe al desbalance del uso y distribución de los recursos y su relación con el entorno (2). Por otra parte, la industria y el comercio han tenido que diversificar sus productos para ampliar la cobertura de sus mercados, ofreciendo productos más pequeños a menor precio, empacados generalmente con materiales cuya vida útil es corta comparada con su proceso de degradación, aumentando así el volumen de manejo de desechos sólidos en las ciudades. Para el manejo integral de estos desechos, el ciclo de tratamiento debe contemplar las siguientes actividades:

1. Control en la generación.

2. Separación de desechos y reciclaje: El Salvador solo recicla 15\% del volumen total.

3. Recolección y transporte: El Salvador tiene una cobertura de recolección de $80 \%$.

4. Tratamiento: Implica la alteración física, química o biológica de los desechos.

5. Disposición final: El Salvador tiene cobertura para disposición final a través de rellenos sanitarios del $78 \%$ del volumen total de desechos y aproximadamente el $10 \%$ de ese volumen total lo ocupa el Tereftalato de Polietileno PET reduciendo la eficiencia y vida útil del sistema de disposición final, porque son plásticos no biodegradables a corto plazo (3).

Para el manejo eficiente de los desechos sólidos se requiere de estrategias tecnológicas para procesar materiales con gran potencial reciclable y que, por sus propiedades y características, puedan convertirse en nuevas materias primas en la fabricación de nuevos productos. Entre estos se destaca el plástico PET que para El Salvador tiene un alto potencial de reciclaje relacionado al valor comercial que posee y a su aporte a la industria del reciclaje mundial (3).

Este hecho no es nuevo para los países de Latinoamérica, pues desde hace más de una década en Argentina se han desarrollado investigaciones para la fabricación de componentes de construcción livianos, de buena aislación térmica, y resistencia mecánica suficiente para cumplir la función de cerramiento lateral de viviendas utilizando materiales plásticos reciclados, promoviendo el uso racional de recursos disponibles en lugar de enterrarlos, quemarlos o acumularlos en basureros al aire libre. (4). En este caso particular de Argentina, el proceso de transformación se logra purificando el polímero reciclado e incorporarlo como agregado fino a la fabricación de ladrillos y bloques prensados mecánicamente, que se endurecen por medio de un proceso de fraguado de la mezcla que es conformada con base de cemento. Si bien es cierto que logra bajar el peso volumétrico con respecto a los bloques y ladrillos del mercado, y la incorporación de polímeros logra bajar la tasa de transferencia de calor en las paredes, la resistencia mínima a la compresión no es alcanzada según la normativa salvadoreña y, por lo tanto, 
la presente investigación descarta utilizar el polímero reciclado como un agregado de elementos de mampostería, aunque se estudiarán las características del polímero PET para conocer sus propiedades y buscar su potencial de transformación después de ser reciclado. Para este fin se detallan las características del polímero. En este sentido, se define al plástico como una sustancia cuya principal característica es la capacidad de ser moldeada bajo diversas circunstancias y creada a partir de la adición de moléculas de carbono para producir otras de gran tamaño, conocidas como polímeros (5).

El tereftalato de polietileno (PET), es el resultado de la polimerización de $1 \mathrm{~mol}$ de tereftalato dimetílico con 2.2 moles de etilenglicol; la primera etapa de polimerización es a $150-200^{\circ} \mathrm{C}$. En la segunda etapa se lleva a cabo un vacío parcial $(0.3 \mathrm{KPa})$ a 260 $290^{\circ} \mathrm{C}$. El grado de cristalización y la dirección de todos los cristalitos rigen las propiedades físicas de la resina. La densidad del PET amorfo es de $1.33 \mathrm{~g} / \mathrm{cm} 3$ en tanto que la densidad de un cristal de PET es de $1.45 \mathrm{~g} / \mathrm{cm} 3$. A temperatura de $70^{\circ} \mathrm{C}$ el material se puede estirar hasta 4 veces su dimensión original, siendo este su estado virgen. Si se calienta nuevamente a más de $100^{\circ} \mathrm{C}$ se vuelve cristalina solo en un $20 \%$ y se vuelve isotrópica a la tensión. Posteriormente se trata a $220^{\circ} \mathrm{C}$ para reacomodar sus partículas, las cuales son $50 \%$ cristalinas, lo que las hace resistente a la tensión. El PET posee una naturaleza químicamente inerte ideal para embalaje de comestibles y medicamentos. La temperatura de fusión es de $255^{\circ} \mathrm{C}$. Generalmente los aditivos que contiene son: Retardadores de llama, tal es el caso de los fosfatos antienvejecimiento, colorantes, agentes de soplado, agentes de cadenas cruzadas y protectores UV. La absorción de luz ultravioleta produce radicales libres que reaccionan con el oxígeno y por ello se produce el fenómeno llamado fotodegradación. Para erradicar este efecto se añaden absorbentes de UV como las Benzofenonas (6).

Para su proceso de reciclaje los embalajes de PET requieren ser separados de la viñeta y de las tapas de Polipropileno (PP), pues estos se funden a menor temperatura y generan cloruro de hidrógeno, el cual cataliza la descomposición del PET. Después de separado, debe ser purificado y triturado para tornarse en nueva materia prima (7).

\section{METODOLOGÍA}

Tomando en cuenta los antecedentes, se decide transformar al PET reciclado con calor considerando su temperatura de fundición a $255^{\circ} \mathrm{C}$ y se fabrican probetas a través de un proceso experimental controlado para ensayos de laboratorio y así obtener resultados que permitieran caracterizar el PET reciclado. Estos ensayos fueron de tipo mecánico y térmico (8). Los ensayos a probetas dieron la pauta para la elaboración de especímenes prototipo de material de construcción que fueron sometidos a otro grupo de ensayos experimentales para la formulación de conclusiones. Se resume a continuación el proceso de fabricación de probetas:

\subsection{Preparación de materia prima con PET reciclado}

El proceso experimental inicia con el reciclaje de 2000 botellas de PET, embalajes para agua y bebidas carbonatadas. Las botellas se purificaron, se secaron a la intemperie, 
se trituraron, transformándose en $47 \mathrm{Kg}$ de PET triturado, en promedio $23.5 \mathrm{~g}$ de hojuelas por botella.

\subsection{Proceso experimental para la fabricación de probetas para ensayos mecánicos y térmicos}

Se realizaron 75 experimentos controlados, tomando los siguientes datos previo a la fabricación de probetas: Peso de la muestra, material del molde, medidas del molde que la contendría, volumen de muestra vaciada en molde, datos de la fuente de calor en la que se fundiría la muestra de material, tiempos de precalentamiento, tiempo de fundición, temperatura de fundición, temperatura de enfriamiento, tiempo de enfriamiento, medidas y peso de los especímenes fundidos, proceso de desmoldado. Habiendo controlado el proceso de fabricación de muestras, se fabricaron las probetas para ensayos mecánicos y térmicos en laboratorio.

\subsection{Proceso de fabricación de especímenes prototipo de materiales de construcción}

Se realizaron dos sesiones de fundición. En la primera se fabricaron tejas curvas plásticas y placas planas cuyas dimensiones son de $15 \mathrm{~cm} \times 30 \mathrm{~cm}$, que son el resultado de vaciar muestras de hojuelas de PET reciclado de $1000 \mathrm{ml}$ en moldes de aluminio, fundidas cada una durante 10 minutos, posteriormente desmoldadas, sometidas a proceso de acabado y pulido de aristas.

La segunda sesión de fundición se realizó en hornos de retemplado de acero, el cual se precalentó a $300^{\circ} \mathrm{C}$ para mantener temperatura constante a $280^{\circ} \mathrm{C}$ por el tamaño de los hornos. La pieza curva que se obtiene es de $35 \mathrm{~cm} x 45 \mathrm{~cm}$. Se fundieron tejas de PET y de PET combinado con PP (material de los tapones), de geometría ondulada, que fueron instaladas en estructuras de madera para ser expuesto a la intemperie.

\section{RESULTADOS}

\subsection{Ensayos térmicos para caracterización del PET reciclado fundido}

Las pruebas térmicas realizadas son: Conductividad térmica, reflectividad, transmisividad, calor específico, dilatación térmica. En el caso de la conductividad térmica, la reflectividad y la transmisividad, se realizaron pruebas antes y después de someter a las probetas a radiación UV, para tratar de predecir la degradación por este efecto. Se proporcionaron 25 probetas planas de $25 \mathrm{~cm} \times 25 \mathrm{~cm}$; a todas ellas se les midió la reflectividad y transmisividad y después se seleccionaron 8 probetas para medir la conductividad. Los criterios de selección fueron dos que tienen relación con el proceso de fabricación de ellas: el primero se refería a las deformaciones sufridas por el método de fabricación, y se calificaron según los siguientes criterios: 1-superficies planas, 2-superficies ligeramente deformadas, 3 -superficies muy deformadas. El segundo criterio se refiere a lo translucido de las probetas, debido a que por la fabricación, estas muestran partes opacas y partes translúcidas. Se calificaron según el siguiente criterio: A-translúcidas, 
B-poco opaca, C-muy opaca. Las probetas seleccionadas para medir la conductividad tienen que cumplir con ser A1, superficies planas y translúcidas.
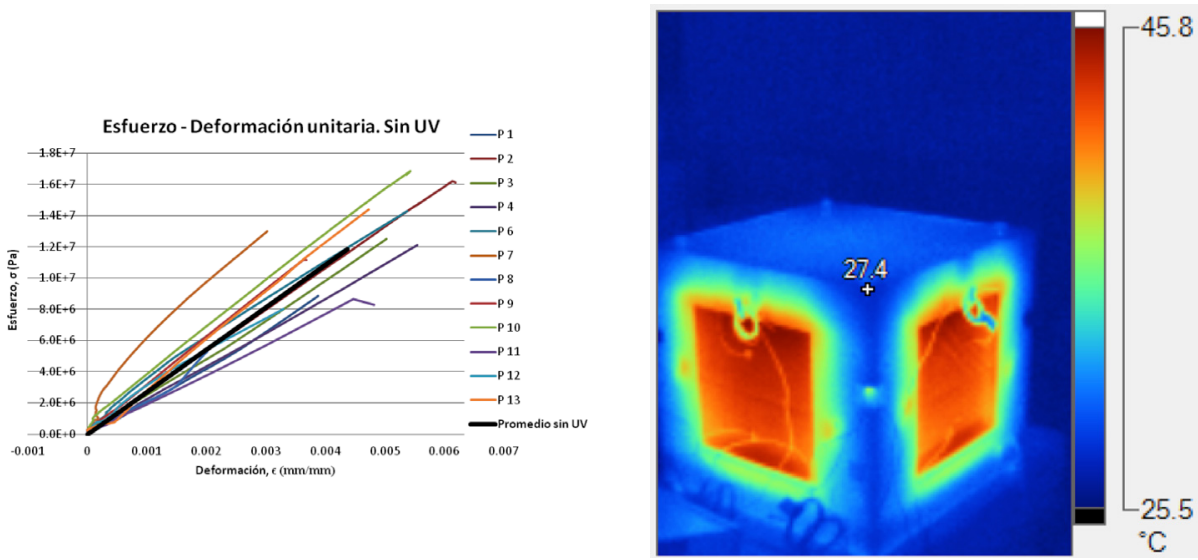

Figura 2. Ensayo para obtener conductividad térmica, termografía.

\subsubsection{Resultados de Conductividad térmica}

La conductividad térmica es una propiedad del material que indica qué tan buen o mal conductor del calor por conducción es el material (9)

$$
\left.\dot{Q}_{\text {cond }}=k * A * \frac{\partial T}{\partial x} \quad \text { (Ecuación de Fourier }\right)
$$

Para el caso, la relación empleada en el cálculo de la conductividad, considerando flujo de calor uniforme y estado estable es:

$$
k=\dot{Q}_{\text {cond }} /\left(A * \frac{\Delta T}{\Delta x}\right)
$$

$k$ : conductividad térmica.

A: $\quad$ área transversal al flujo de calor.

$\Delta T: \quad$ diferencia de temperatura entre las caras de la probeta.

$\Delta x$ : espesor de la probeta.

Qcond: calor por conducción

La conductividad medida para las probetas proporcionadas es:

\begin{tabular}{lll}
\hline Promedio antes de UV & $0.12 \pm 0.01$ & W/m-K \\
\hline
\end{tabular}

\subsubsection{Resultado de Reflectividad (reflectancia)}

La reflectancia es propiedad de una superficie (material, rugosidad, etc.) e indica qué fracción de energía incidente refleja dicha superficie (9). Esta prueba se realiza sobre 
las dos caras de 25 probetas, ya que estas presentan diferencias debido a marcas de los moldes de fabricación, llamando cara A a la superficie libre y cara B a la superficie en contacto con el molde. Sobre cada una de las caras de cada probeta se hace incidir cierta cantidad de energía radiante midiendo la energía radiante que esta refleja, y la relación entre estas energías es la reflectividad del material.

$$
\rho=\frac{\text { Energía reflejada }}{\text { Energía incidente }}
$$

La reflectividad medida en las probetas proporcionadas es:

\begin{tabular}{|c|c|c|c|}
\hline \multicolumn{2}{|c|}{ Categoría } & \multicolumn{2}{|c|}{ Antes de UV } \\
\hline $\mathrm{C} 1$ & $\mathrm{C} 2$ & Lado A & Lado B \\
\hline \multicolumn{2}{|c|}{ Promedio } & $10.87 \pm 1.53$ & $10.61 \pm 1.53$ \\
\hline
\end{tabular}

En promedio la cara A refleja el $10.87 \%$ (reflectancia=0.11) de la luz incidente, y la cara B el $10.61 \%$ (reflectancia $=0.11$ ), no hay diferencia entre las dos caras.

\subsubsection{Resultado de Transmisividad (transmitancia)}

La transmitancia es una propiedad del material y el espesor de la muestra; en este caso, las probetas tienen un espesor promedio de $0.00324 \mathrm{~m}$. Para medir la transmitancia, se hace incidir energía radiante por una de sus caras y se mide cuánta de esta radiación logra atravesar a la muestra, la relación entre la energía transmitida y la energía incidente (9).

$$
\tau=\frac{\text { Energía transmitida }}{\text { Energía incidente }}
$$

En este caso se espera gran diferencia de los valores de transmisividad entre las probetas calificadas como A, B y C. Para el caso, se debe prestar atención a las catalogadas como A, ya que son las que representan el producto deseado. En promedio las muestras

\begin{tabular}{|c|c|c|}
\hline \multicolumn{2}{|c|}{ Categoría } & \multirow[b]{2}{*}{ Antes de UV } \\
\hline $\mathrm{C} 1$ & $\mathrm{C} 2$ & \\
\hline \multicolumn{2}{|c|}{ Promedio } & $34.57 \pm 5.00$ \\
\hline
\end{tabular}
catalogadas tipo A transmiten el 34.57\% (transmisividad = 0.35) de la energía incidente.

\subsubsection{Resultado de Absortividad (absortancia)}

La absortividad es una propiedad del material y del espesor de este (9). En este caso, 
las probetas tienen un espesor promedio de $0.00324 \mathrm{~m}$. Las muestras deben de cumplir con la relación: Transmitancia + reflectancia + absortancia $=1,(\tau+\varrho+\alpha=1)$ ya que se utiliza la misma fuente; por lo tanto la absortancia es:

\begin{tabular}{ll}
\hline Absortancia & $54.56 \pm 15.28$ \\
\hline
\end{tabular}

Como es un valor indirecto, que se obtiene midiendo dos propiedades distintas, el valor tiene una gran incertidumbre $(28 \%)$ En promedio las muestras catalogadas tipo A absorben el 54.56\% (absortividad $=0.55$ ) de la energía incidente.

\subsubsection{Resultado de muestras sometidas a UV}

Las probetas catalogadas como A1 fueron sometidas a radiación UV para observar su efecto sobre sus propiedades de transmisividad, reflectividad y absortividad. Se tomaron tres series de datos, los datos iniciales después de 40 días fueron equivalentes de exposición a rayos UV llamados intermedios, y los datos finales tomados a los 78.25 días, fueron equivalentes. En los primeros 40 días las muestras recibieron 16,097 kJ/ m2 de energía UV en el rango de longitud de onda $320 \mathrm{~nm}-400 \mathrm{~nm}$, y en los 78.25 días equivalentes recibieron $31,483 \mathrm{~kJ} / \mathrm{m} 2$ de radiación $\mathrm{UV}$ en el rango mencionado.

Tabla 1: Variaciones de datos en ensayos térmicos.

\begin{tabular}{lccccccc}
\hline & $\begin{array}{c}\text { Medida } \\
\text { inicial }\end{array}$ & $\begin{array}{c}\text { Medida } \\
\text { intermedia }\end{array}$ & Variación & $\begin{array}{c}\text { Variación } \\
\text { porcentual }\end{array}$ & $\begin{array}{c}\text { Medida } \\
\text { final }\end{array}$ & Variación & $\begin{array}{c}\text { Variación } \\
\text { porcentual }\end{array}$ \\
\hline Conductividad (W/m-K) & 0.12 & 0.10 & 0.02 & 16.67 & 0.07 & 0.05 & 41.67 \\
Reflectividad & 0.11 & 0.07 & 0.04 & 36.36 & 0.07 & 0.04 & 36.36 \\
Transmisividad & 0.35 & 0.40 & -0.05 & -14.29 & 0.40 & -0.05 & -14.29 \\
Absortividad & 0.54 & 0.53 & 0.01 & 1.85 & 0.53 & 0.01 & 1.85 \\
\hline
\end{tabular}

Fuente: Elaboración propia

\subsubsection{Resultado de ensayo de calor específico}

El calor específico es una propiedad del material y se define como la cantidad de calor que hay que añadir a la unidad de masa del material para que este cambie su temperatura en 1 grado de temperatura. $\mathrm{c}=\frac{\mathrm{Q}}{\mathrm{m} * \Delta \mathrm{T}}$

$$
\mathrm{m}_{\text {agua }} \mathrm{c}_{\text {agua }}\left(\mathrm{T}_{\text {equilibrio }}-\mathrm{T}_{\mathrm{i} \text { agua }}\right)=\mathrm{m}_{\text {probeta }} \mathrm{c}_{\text {probeta }}\left(\mathrm{T}_{\text {i probeta }}-\mathrm{T}_{\text {equilibrio }}\right)
$$

El calor específico de las probetas es:

\begin{tabular}{lll}
\hline calor específico & $1.03 \pm 0.11 \quad \mathrm{~kJ} / \mathrm{kg}-\mathrm{K}$ \\
\hline
\end{tabular}




\subsection{Ensayos mecánicos para caracterización del PET reciclado fundido}

El ensayo a tensión se realizó en la máquinaUIB-1000-W a una velocidad de $5 \mathrm{~mm} / \mathrm{min}$ Se colocó el extensómetro en el área destinada para ello. En la "Longitud Calibrada", se utilizó un micrómetro con un error máximo de $0.0002 \mathrm{~mm} / \mathrm{mm}$ como lo indica la Norma ASTM D638-10. Se probó un lote de probetas en dos etapas; la primera, recién fabricadas y la otra, después de haber sido expuestas a luz ultravioleta. Para conseguir una mayor exposición equivalente a un mayor tiempo de vida, se colocaron las probetas en una cámara de radiación UV $12646.71 \mathrm{~kJ} / \mathrm{m}^{2}$ por 31 días. Se analizó la radiación UV por ser uno de los factores que más inciden en la durabilidad y es al que más está expuesta una cubierta. No se controló la humedad, sino que se dejaron expuestas al medio ambiente, ya que esas serán sus condiciones normales de funcionamiento. De las pruebas citadas anteriormente, la prueba D638 (10) proporciona el mínimo de datos necesarios para un diseño estructural preliminar. La cantidad de muestras recomendadas por la norma ASTM D638-10 "sección 7.1" es de 5 muestras para materiales isotrópicos (material que posee las mismas propiedades físicas en todas las direcciones). Sin embargo, para aumentar la confiabilidad de los datos se tomaron 12 pruebas para cada grupo. Las gráficas a continuación, Fig. 3 y 4, resumen los comportamientos de las probetas en cada uno de los grupos. La línea identificada en cada una de ellas como "promedio" es la línea calculada correspondiente de la media estadística de módulo de elasticidad, esfuerzo de resistencia a la tensión y a la ruptura.

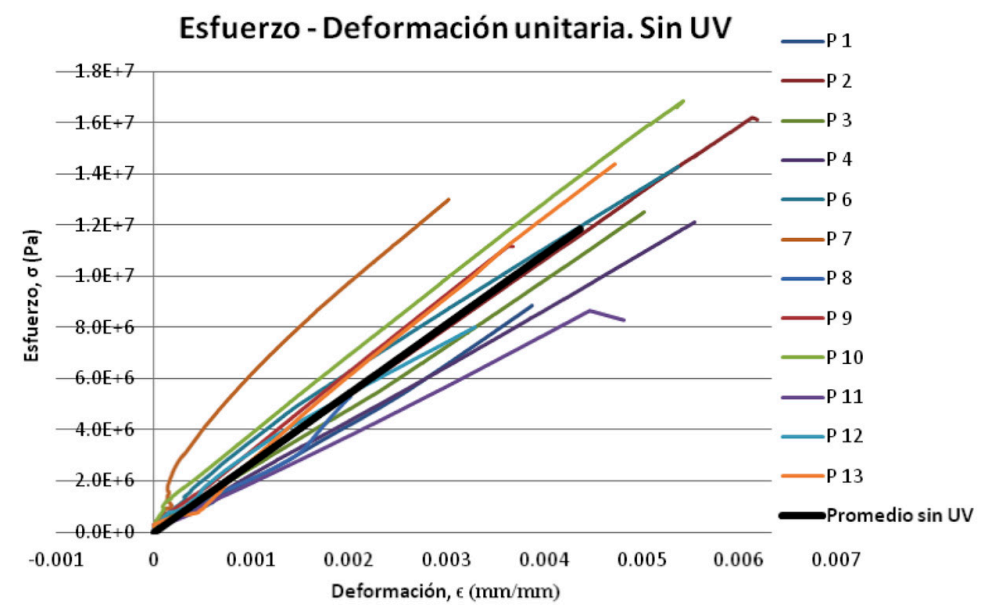

Figura 3. Esfuerzo versus deformación unitaria en probeta sin exposición a radiación UV

Se obtuvo una media aritmética de 11.874 MPa luego de depurar los resultados con la desviación típica obtenida de 3.498 MPa. Según la Norma ASTM D 638-10 "sección 11.7" Los resultados se resumen en la Tabla 2 y fueron obtenidos a través de las lecturas directas del sensor y del extensómetro. 


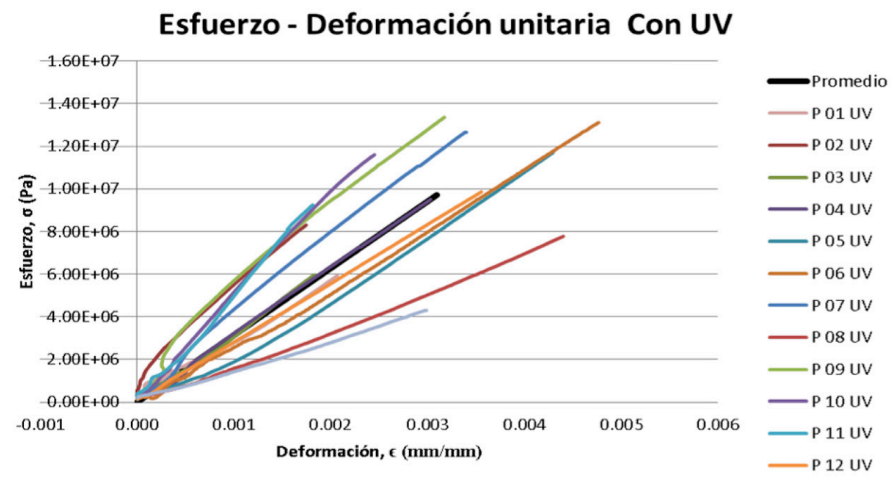

Figura 4. Esfuerzo versus deformación unitaria por probeta con exposición a radiación UV.

Tabla 2. Media aritmética y desviación típica obtenidos por grupos, con y sin exposición a radiación UV en MPa

\begin{tabular}{lcccccc}
\hline & \multicolumn{3}{c}{ Sin UV } & \multicolumn{3}{c}{ Con UV } \\
\cline { 2 - 6 } & $\begin{array}{l}\text { Módulo de } \\
\text { Elasticidad }\end{array}$ & $\begin{array}{l}\text { Resistencia } \\
\text { de Tensión } \\
\text { a la ruptura }\end{array}$ & $\begin{array}{l}\text { Límite de } \\
\text { Extensión }\end{array}$ & $\begin{array}{c}\text { Módulo de } \\
\text { Elasticidad }\end{array}$ & $\begin{array}{l}\text { Resistencia } \\
\text { de Tensión } \\
\text { a la ruptura }\end{array}$ & $\begin{array}{l}\text { Límite de } \\
\text { Extensión }\end{array}$ \\
\hline Media Aritmética [MPa] & 2708.01 & 11.87 & 10.55 & 3137.76 & 9.71 & 7.93 \\
$\begin{array}{l}\text { Desviación Típica [MPa] } \\
\begin{array}{l}\text { Cambio en el valor } \\
\text { promedio[\%] }\end{array}\end{array}$ & 807.80 & 3.49 & 5.026 & 1299.77 & 2.94 & 4.84 \\
\hline
\end{tabular}
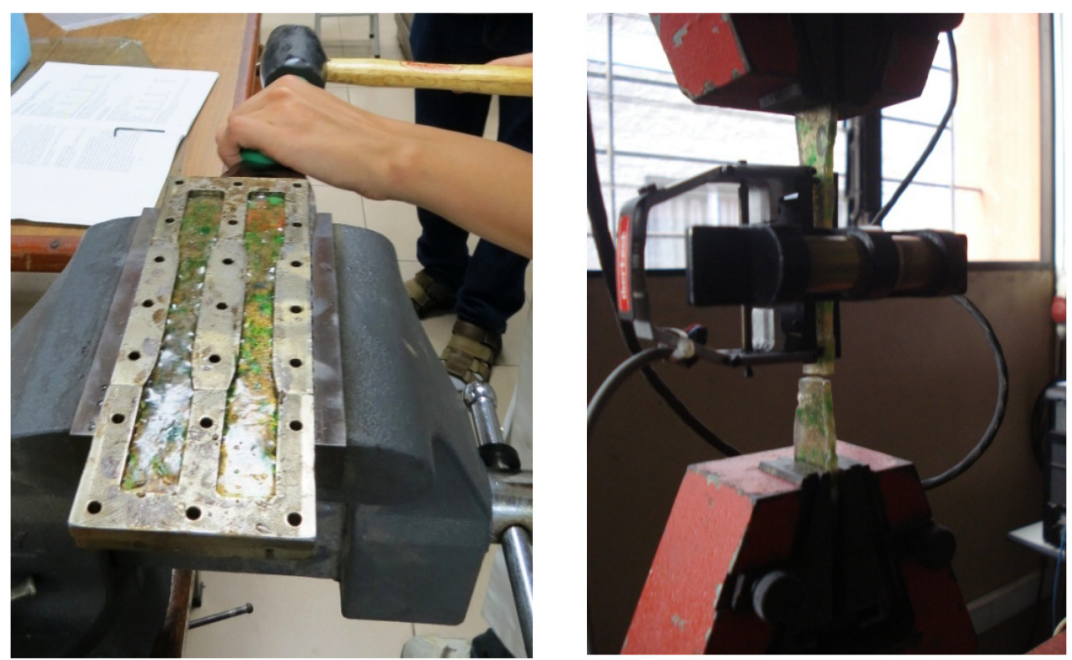

Figura 5. Elaboración de probetas y ensayo a tensión de probetas. 


\subsection{Ensayos experimentales para la caracterización de las tejas de PET}

\subsubsection{Ensayo de densidad}

Se realizaron cuatro ensayos para obtener la densidad del PET reciclado con un coeficiente de variación estándar menor al 5\%, se obtienen los siguientes datos:

- Densidad promedio de PET reciclado sin fundir en hojuela $0.4346 \mathrm{~g} / \mathrm{cm}^{3}$

- Densidad promedio de PET reciclado fundido $1.2703 \mathrm{~g} / \mathrm{cm}^{3}$

\subsubsection{Medición de emisiones en fundición de plástico}

Para determinar si el PET reciclado fundido presentaba emisiones de Dióxido de Carbono se fundieron dos muestras de $500 \mathrm{ml}$, con un peso de $231.72 \mathrm{~g}$ cada una, en presencia del detector de gases tóxicos modelo 8014KA y no se observó reacción en el dispositivo para $\mathrm{CO}_{2}$, por lo tanto se estima que no hay presencia de Dióxido de Carbono en la fundición realizada.

\subsubsection{Medición de consumo de energía eléctrica en fundición de plástico.}

Para este ensayo se realizó la fundición de dos muestras de $750 \mathrm{ml}$ con un peso de 279.60g cada una; se procedió a conectar el medidor de potencia Fluke modelo 43-B al tablero que alimenta la fuente de calor, se realizó una primera medición para determinar el consumo de energía durante el periodo de precalentamiento; luego, se ingresa una muestra de PET reciclado y se toma la medida del consumo de energía durante el tiempo de fundición de la probeta. Este procedimiento se realizó dos veces. La fuente de calor tardó 26 minutos en alcanzar los $280^{\circ} \mathrm{C}$ y reportó $4000 \mathrm{KW}$. Se pudo observar que mientras no hubo cambio de temperatura, el consumo fue menor entre 1200-2000 KW y mientras la compuerta de la fuente de calor no estuvo abierta, el consumo fue nulo.

\subsubsection{Ensayo de absorción}

El porcentaje de absorción se obtiene primero restando peso saturado menos peso seco; luego, se divide este valor entre el peso seco. Los porcentajes varían entre $0.11 \%$ y $1.77 \%$ reflejando un promedio bajo de absorción; se estima que el material fundido no es absorbente, sino impermeable.

\subsubsection{Ensayo al impacto}

Para este ensayo se dividió el grupo de tejas a ensayar en dos lotes con características similares. El primer ensayo consistió en dejar caer sobre el espécimen una bola de acero dentro de un tubo de acero de $25 \mathrm{~cm}$ de longitud para medir resistencia al impacto. Para el lote A, la masa 1: 200.4g y para lote B, la masa 2: 355.2g. Se realizó esta prueba a los ocho especímenes de teja, de los cuales ninguno presentó planos de falla. Para el segundo ensayo se cambió la altura de caída de la masa, aumentándola a 100cm. En este ensayo, el lote A con masa 1 presentó pequeños planos de falla, mientras en el lote B con masa 2 , tres de cuatro especímenes colapsaron con el impacto. Considerando que la masa 2 es 1.8 veces mayor que la masa 1, pudo observarse que al realizar la prueba de 
impacto a $25 \mathrm{~cm}$, ninguno de los especímenes sufrió daños, pero al generarle caída libre a un metro de altura con los dos tipos de masas, las tejas rompen de $355.2 \mathrm{~g}$, excepto por la teja combinada de PET con PP que no sufre ningún tipo de daño.

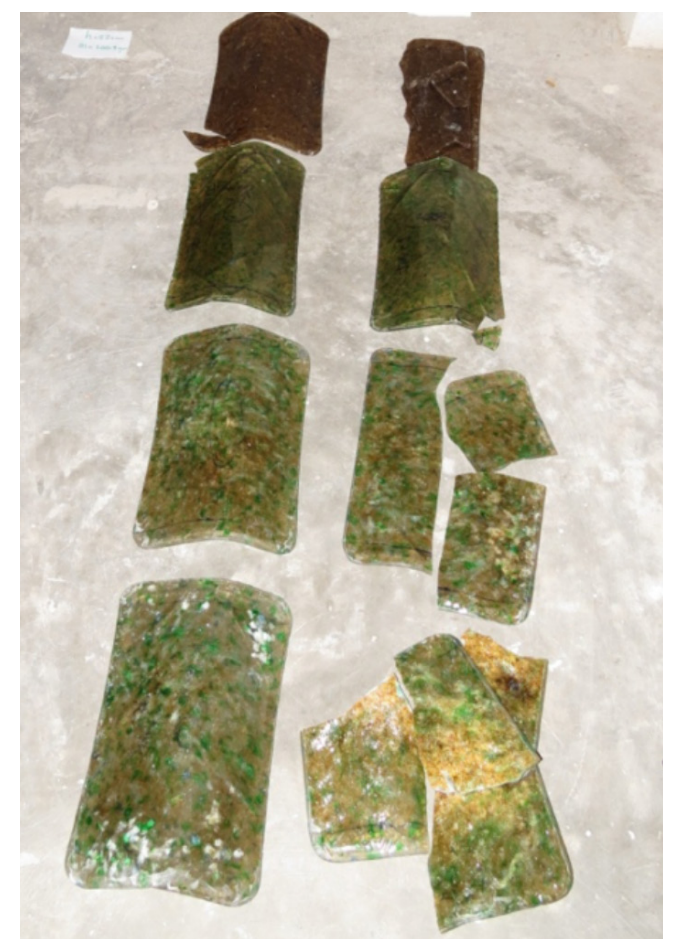

Figura 6. Resultado del ensayo al impacto.

\subsubsection{Ensayo de carga dinámica}

Este ensayo se desarrolló bajo el principio de brazo de palanca. Como primer punto se procedió a ajustar el espécimen en uno de los extremos de la máquina y a los puntos de apoyo de la misma. Posteriormente, se colocó en el extremo opuesto de la máquina un recipiente, cuya masa se despreció, agregándole peso gradualmente. Se comparó con otros materiales, y las tejas elaboradas a partir de PET reciclado soportaron dos veces más que las tejas de micro-concreto y cuatro veces más que la teja de arcilla. Puede destacarse que el lote ha soportado una carga máxima de $16.3 \mathrm{Kg}$ en 30 minutos de aplicación de carga.

\subsubsection{Ensayo a flexión en tejas sin exposición a intemperie}

Se colocó el espécimen en la máquina universal, ajustando los apoyos y el punto de transferencia de carga en el espécimen, debido a su forma cóncava. Posteriormente, se aplica la carga al espécimen hasta su punto de ruptura. Este proceso se realizó con tres especímenes de teja, dos de ellos de PET reciclado y uno de PET combinado con PP, siendo este último el único que se deformó hasta formar fisura con una carga de $200 \mathrm{Kg}$. Los otros dos especímenes rompieron con una carga de $120 \mathrm{Kg}$ y $160 \mathrm{Kg}(11,12)$. 


\subsubsection{Ensayo a flexión en tejas expuestas a intemperie por 14 meses}

Se colocó el espécimen en la máquina universal, ajustando los apoyos y el punto de transferencia de carga en el espécimen, debido a su forma cóncava. Posteriormente se aplica la carga al espécimen hasta su punto de ruptura. Este proceso se realizó con 18 especímenes de teja de PET con un promedio de carga de ruptura de $99.0 \mathrm{Kg}$, siendo este mayor comparado con la carga de ruptura de la teja de barro de $60 \mathrm{Kg}$. Se calculó un error típico de 5.57, una desviación estándar de $23.64 \mathrm{Kg}$ y un nivel de confianza del 95\% $(11,12)$.
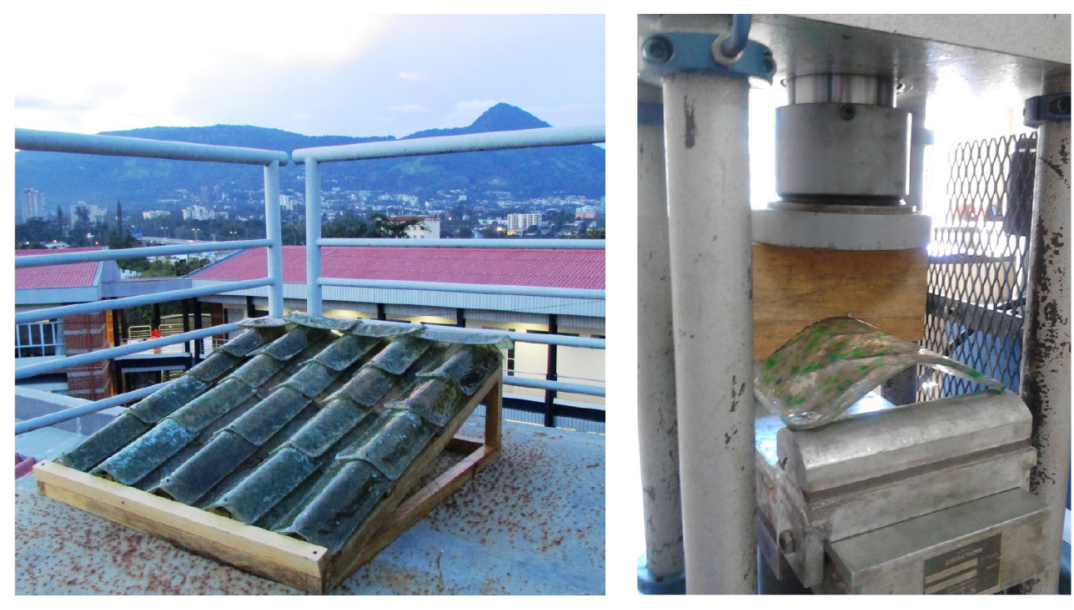

\section{DISCUSIÓN DE RESULTADOS}

Las investigaciones precedentes no reflejan datos que aporten a la caracterización del PET reciclado como materia prima que a través de un proceso de transformación controlado pudiera convertirse en nueva opción de material para la construcción; por lo tanto, la presente investigación es un aporte que sienta las bases sobre cómo transformar el PET reciclado con calor de forma no industrializada con valores de resistencias tanto mecánicas como térmicas que cumplen con lo estipulado en el reglamento de construcción, para que pueda ser considerado un nuevo material de construcción para cubierta y revestimientos. Se enumeran las siguientes conclusiones que comprueban la discusión:

1. El Reglamento para la Seguridad Estructural de las Construcciones en El Salvador RESESCO establece que para elementos de techo cuya pendiente sea mayor que el 5\% deberán revisarse con una carga concentrada de $100 \mathrm{Kg}$ en la posición más crítica. Por otra parte, los especímenes de tejas sometidos a carga concentrada fallaron con cargas de $120 \mathrm{Kg}$ (el lote fabricado con PET sin purificar); otro grupo falló a $160 \mathrm{Kg}$ (el lote fabricadas con PET purificado) y los especímenes que fallaron a $200 \mathrm{Kg}$ pertenecen al lote fabricado con la combinación de PET con PP purificados. A pesar que todos los lotes presentan un valor de falla mayor a la carga admisible por el reglamento, el fallo no es súbito ya que presenta cierta deformación previa, 
siendo este aspecto muy positivo, considerando su desempeño como cubierta. No obstante, el lote de tejas fabricadas sin purificar ha fallado de forma súbita. Se deduce que la fragilidad y perdida de resistencia del material se debe a la presencia de restos de lixiviados no eliminados por completo en el proceso de preparación de materia prima.

2. El PET reciclado ha presentado una resistencia a la tensión en promedio de 11.7 Mpa equivalentes a $119.34 \mathrm{Kg} / \mathrm{cm}^{2}$. Esta resistencia es un indicador del potencial que el nuevo material tiene para desempeño como material de cubierta; sin embargo, no puede asegurarse que su integridad estructural se mantenga en el tiempo con la exposición a radiación, ya que en el ensayo a tensión del segundo grupo de probetas después de haber sido expuestas a radiación simulada de UV por 30 días, la resistencia disminuyó en un 18\% y aumentó su rigidez en un $15.9 \%$.

3. Además de garantizar la seguridad e integridad de las edificaciones por medio de sus materiales y sistemas constructivos, debe garantizarse el bienestar de las personas que habitan en esas edificaciones. Por lo tanto, se ha buscado conocer datos de variables térmicas de transferencia de calor y así verificar si el material tiene potencial térmico. Se encontró que el material es medianamente conductivo, comparado con los materiales para cubiertas que existen en el mercado. No obstante es 1.5 veces más conductivo que la arcilla, 2 veces menos conductivo que las fibras aglutinadas con cemento y 50 veces menos conductivo que el acero. Esto genera una perspectiva de uso que garantiza que la transferencia de calor se realiza de forma lenta, a razón de $0.12 \mathrm{~W} / \mathrm{mK}$.

4. Los procesos de ensayo han dejado en evidencia que el plástico PET reciclado, al ser expuesto a temperatura mayor a su punto de fusión y a radiación UV prolongada, sufre un proceso de degradación a corto plazo, el cual no garantiza la durabilidad mínima de 5 años, comparada con las garantías que brindan otros productos en el mercado para cubiertas, ya que la disminución de sus propiedades se atribuye a la degradación que el material sufre en presencia de rayos UV.

\section{REFERENCIAS}

1. Cisneros A, Rodríguez L. Tecnología en Construcción. El Salvador. 1ª ed. UCA Editores. UCA El Salvador; 2006.

2. Programa Nacional para el Manejo Integral de los Desechos Sólidos. Plan para el Mejoramiento del Manejo de Desechos Sólidos en El Salvador. El Salvador: Gobierno de El Salvador; 2010.

3. Meléndez E. Estudio sobre el Mercado Potencial del Reciclaje en El Salvador. $1^{\mathrm{a}}$ ed. El Salvador: Ministerio del Ambiente y Recursos Naturales; 2006.

4. Gaggino R. Ladrillos y placas prefabricadas con plásticos reciclados aptos para la autoconstrucción Revista INVI. 2008;23(63):137-63. Disponible en: https://es.scribd.com/ document/250525682/Ladrillos-y-placas-prefabricadas-con-plasticos-reciclados-Gaggino-pdf

5. García S. Referencias históricas y evolución de los plásticos. España: Universidad Politécnica de Valencia; 2008.

6. Harper C, Modern Plastics. Manual de Plásticos, $1^{\mathrm{a}}$ ed. Vol. 1. México: McGraw-Hill Interamericana; 2004. 
7. Harper C, Modern Plastics. Manual de Plásticos, $1^{\mathrm{a}}$ ed. Vol. 2. México: McGraw-Hill Interamericana; 2004.

8. De Cusa J. Aplicaciones del plástico en la construcción. Barcelona, España: CEAC; 1979.

9. Cengel, Y.A. Transferencia de calor. México: McGraw-Hill; 2004.

10.ASTM Standard D638-10. Standard Test Method for Tensile Properties o Plastics. USA: ASTM International; 2010.

11. ASTM Standard C1167-11. Standard Specification for Clay Roof Tiles. USA: ASTM International; 2011.

12. ASTM Standard C67-14. Standard Test Methods for Sampling and Testing Brick and Structural Clay Tile. USA: ASTM International; 2014. 